\title{
Intra-arterial interventional therapy for inoperable local advanced breast cancer: A retrospective study
}

\author{
JING MA, JIN SONG, HAI CHEN, CIBO FAN, JIAQI XIE and XIAODONG QI \\ Department of Breast Surgery, People's Liberation Army Army General Hospital, Beijing 100700, P.R. China
}

Received November 22, 2016; Accepted October 13, 2017

DOI: $10.3892 / \mathrm{ol} .2017 .7507$

\begin{abstract}
The aim of the present study was to evaluate the safety and efficacy of intra-arterial interventional therapy (IAIT) in the treatment of inoperable local advanced breast cancer (LABC). A total of 7 patients with pathologically proven inoperable LABC were included in the present study. Patients received 1-4 cycles of IAIT prior to mastectomy and postoperative adjuvant therapy. The safety and clinical outcomes of IAIT were retrospectively analyzed. Between February 2009 to September 2016, 7 patients received IAIT. The youngest patient was 34 years old and the eldest was 90 years old. The tumor size ranged between 6 and $20 \mathrm{~cm}$ in diameter. A total of 5 patients presented with palpable lymph nodes, while none of the patients exhibited distant metastatic disease. A total of 6 patients received $\geq 1$ cycle of neoadjuvant chemotherapy prior to IAIT and no severe side effects were observed. Overall, 6 patients exhibited a partial response and 1 presented with stable disease following treatment. The range of progression-free survival was 6-88 months. In total, 1 patient succumbed as a result of another disease 8 months after IAIT, another succumbed from carcinoma of the right fallopian tube and multiple organ metastases 9 months after IAIT, and another survived for 11 months and died of heart disease after IAIT. The other 4 patients remain alive. IAIT is safe and effective for patients with inoperable LABC, and thus, may be an appropriate alternative for patients who are not responsive to or are unable to tolerate neoadjuvant chemotherapy.
\end{abstract}

\section{Introduction}

Breast cancer is one of the most common malignancies in females, particularly in developed countries, including those of Europe and the USA. Each year, $\sim 1$ million females will

Correspondence to: Dr Xiaodong Qi, Department of Breast Surgery, People's Liberation Army Army General Hospital, 5 Nanmencang, Dongcheng, Beijing 100700, P.R. China

E-mail: s2006134@126.com

Key words: intra-arterial interventional therapy, local advanced breast cancer, neoadjuvant chemotherapy, modified radical mastectomy, radiotherapy, endocrinotherapy be diagnosed with breast cancer globally (1). The incidence of breast cancer has steadily increased over previous years, with patients being affected at a much younger age, which poses a serious health threat with a large global impact (2). In 2014, 232,670 females were diagnosed with breast cancer and 40,000 fatalities resulted from it in the USA (3). Although the incidence of breast cancer is lower in China, it is increasing annually in urban and economically developed areas (4). With the development of methods for the early detection of breast cancer and novel agents for chemotherapy, endocrinotherapy and targeted therapy, early-stage breast cancer has been well controlled. However, the treatment of advanced-stage breast cancer has been less successful. The National Comprehensive Cancer Network guidelines define locally advanced breast cancer (LABC) as Tumor-Node-Metastasis (TNM) stage IIIA [T0-2, N2, M0; T3, N1-2, M0], stage IIIB (T4, N0-2, M0) or IIIC (T0-4, N3, M0) (5). Surgical resection is the most effective method for treating operable LABC (clinical stage T3, N1, M0). For inoperable LABC, preoperative neoadjuvant chemotherapy with anthracycline alone or combined with taxane is the standard treatment. If patients are human epidermal growth factor receptor 2 (HER2)-positive, trastuzumab is used simultaneously. If the patients are responsive to chemotherapy, surgical resection, radiotherapy or endocrinotherapy may follow. For patients with disease progression during preoperative chemotherapy, palliative breast irradiation is advised to enhance local control. Subsequent systemic therapy is also standard. However, if patients are not responsive to any of these treatments, intra-arterial interventional therapy (IAIT) may serve as a safe and effective alternative for the management of inoperable LABC. The present study aimed to evaluate the safety and efficacy of intra-arterial interventional therapy (IAIT) in the treatment of LABC.

\section{Patients and methods}

Patients. The inclusion criteria of the present study were as follows: Patients with pathologically-proven inoperable LABC who were not suitable for mastectomy following primary treatment. The exclusion criteria were as follows: Patients who had severe coagulation mechanism abnormality or had severe cardiac, renal or liver failure. A total of 7 patients were enrolled in the present study between February 2009 and September 2016 (Table I). All patients volunteered to participate in the program and provided written informed consent. Overall, 
1 patient did not tolerate neoadjuvant chemotherapy, 2 were partially responsive to neoadjuvant chemotherapy but were unsuitable for mastectomy, 2 presented with disease progression, 1 exhibited stable disease, and a 90-year-old patient was in a poor physical condition and was unable to tolerate neoadjuvant chemotherapy. All the patients received IAIT-centered systemic treatment. All IAIT was performed by senior doctors in the Department of Interventional Radiology at the People's Liberation Army Army General Hospital (Beijing, China).

IAIT. All 7 patients were cannulated via the femoral artery ipsilateral to the lesion. Subsequently, a $4 \mathrm{~F}$ fixed-curve catheter (Cobra catheter; Cook Medical, Inc., Bloomington, IN, USA) was advanced into the internal mammary artery ipsilateral to the lesion and into the subclavian artery. Digital subtraction angiography (DSA) was performed to determine the blood supply to the tumor. IAIT with or without embolization was subsequently administered to the patients every 3 weeks on average. The regimens for IAIT were different. During the early years our breast surgeons posed the idea of IAIT, while the IAIT was performed by doctors in the interventional radiology department in People's Liberation Army Army General Hospital. So what regimen should be used was almost decided by them. And the regimen was the most often used for solid tumors in their practice, which proved effective. In recent years, with the deep understanding and treatment of breast cancer, especially with the worldwide accept of NCCN guidelines, anthracycline combined with or without taxane become the standard treatment. So now the doctors in the interventional radiology department accept our advice to use anthracycline and taxane based regimens for IAIT.

Due to space limitations, only four representative cases were listed below.

Case 1. An 83-year-old woman visited the Department of Breast Surgery, People's Liberation Army Army General Hospital in February 2009 due to a tumor with a walnut-like appearance on the left breast that had become apparent 6 years previously. The tumor was not painful and therefore the patient had not consulted a doctor or undergone any prior treatment. After 4.5 years, the tumor had grown larger and became ulcerated with bleeding. The patient attended a local hospital in order to attenuate the bleeding, but no other treatments were provided. By the time the patient attended the People's Liberation Army Army General Hospital, the tumor was $15 \times 15 \mathrm{~cm}$ in diameter with a cauliflower-like appearance (Fig. 1). Skin ulceration, necrosis, bleeding and effusion were observed, along with an unpleasant odor. The tumor was fixed to the chest wall and was not movable. The patient presented with severe anemia and heart failure, with a hemoglobin level of $32 \mathrm{~g} / \mathrm{l}$ (normal range: 110-150 g/l) and a hematocrit of 0.11 (normal range: 0.37-0.47). Anemia and heart failure were corrected through blood transfusion, wound dressing was conducted daily in order to improve the local condition and antibiotics were administered in order to control the lung infection. Biopsy revealed a neuroendocrine carcinoma and immunohistochemistry revealed the following: weakly positive estrogen receptor (ER) and progesterone receptor (PR) expression, and HER2-negative. The patient was administered IAIT (30 mg pirarubicin, $30 \mathrm{mg}$ hydroxycamptothecin and
$250 \mathrm{mg}$ fluorouracil) via the left internal thoracic artery and the subscapular artery. Simultaneous intra-arterial embolization with selective absorbable gelatin sponge particles was conducted. Following the first cycle of IAIT, the tumor decreased to a diameter of $8 \times 7 \mathrm{~cm}$ in diameter. During the second and third cycles of IAIT, $20 \mathrm{mg}$ pirarubicin, $15 \mathrm{mg}$ hydroxycamptothecin and $500 \mathrm{mg}$ fluorouracil were administered. Following 3 cycles of IAIT, the tumor measured $5 \times 4 \mathrm{~cm}$ in diameter (Fig. 2). In May 2009, the patient underwent a modified radical mastectomy. Pathology identified neuroendocrine carcinoma and carcinoma emboli in the vessels. One axillary lymph node exhibited invasion by the carcinoma (1/18). Immunohistochemistry revealed that the tumor was ER-positive (90\%), PR-positive (90\%), HER2-negative and Ki-67-positive (5\%). The patient recovered from the surgery without complications (Fig. 3) and accepted endocrinotherapy (exemestane $25 \mathrm{mg}$ once daily) for 6 months afterwards, but refused any subsequent treatment. The patient is now 90 years old and remains healthy.

Case 2. A 90-year-old woman attended the Department of Breast Surgery, People's Liberation Army Army General Hospital in January 2013. In April 2012, the patient found a $3-\mathrm{cm}$ tumor of the left breast. The tumor was hard and painful when palpated. The patient was diagnosed with breast cancer at another hospital clinically without a biopsy being performed, and due to an advanced age, did not receive any treatment. The tumor grew to $\sim 6 \mathrm{~cm}$ in diameter and the surrounding skin became red, and the patient subsequently attended the same hospital again in December 2012. A tumor biopsy revealed invasive ductal carcinoma and immunohistochemistry revealed that the tumor was ER-negative, PR-negative, HER2-negative and Ki-67-positive (70\%). Administration of paclitaxel liposome neoadjuvant chemotherapy (90 mg on days 1,8 and 15) was planned, however, due to serious side effects, including nausea, vomiting and fever, the patient did not complete the first cycle. When the patient attended the People's Liberation Army General Hospital, the tumor was $6 \mathrm{~cm}$ in diameter with a 16x15 cm are of surrounding red skin (Fig. 4). The tumor was hard and stiff without a distinct boundary, and it was fixed to the chest wall and immovable. The patient was administered IAIT (30 mg pirarubicin and $120 \mathrm{mg}$ paclitaxel) twice via the left internal thoracic artery and the subscapular artery. Subsequently, 2 cycles of IAIT with $40 \mathrm{mg}$ epirubicin and $150 \mathrm{mg}$ paclitaxel, and intra-arterial embolization with selective absorbable gelatin sponge particles, was performed. Following 4 cycles of IAIT, the tumor diameter decreased to $\sim 4 \mathrm{~cm}$ and the skin returned to an almost normal color. The tumor became soft and movable with a clear boundary. The patient tolerated IAIT well and no serious side effects were observed. In May 2013, a modified radical mastectomy was performed. Pathology revealed aninvasive ductal carcinoma that was ER-negative, PR-negative, HER2-negative and Ki-67-positive (30-60\%). Carcinoma emboli were observed in the vessels. The axillary lymph nodes (10/10) and the subclavian lymph nodes (7/7) exhibited invasion by the carcinoma. The patient recovered normally without complications (Fig. 5), but succumbed to another disease in September 2013. 


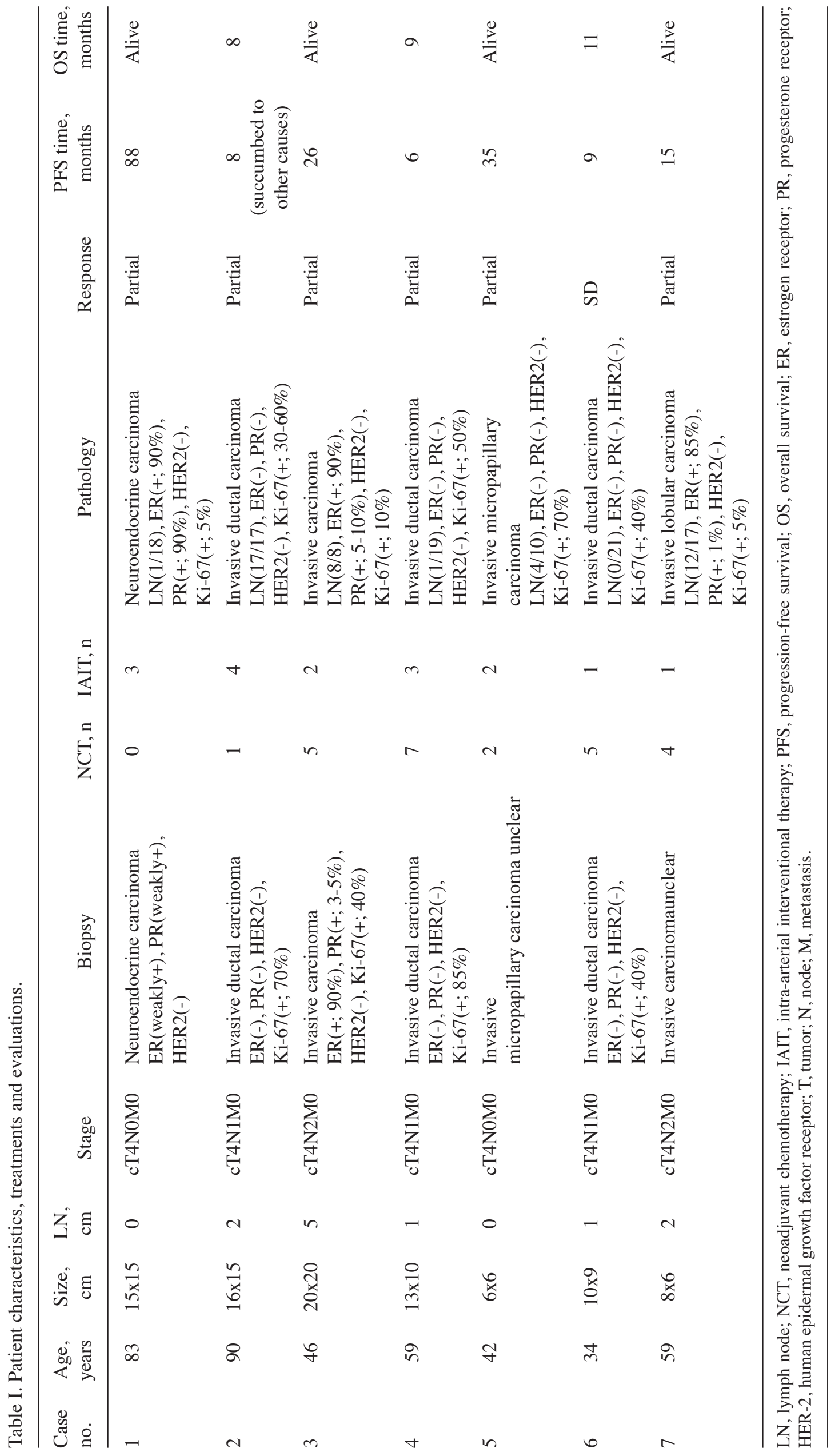




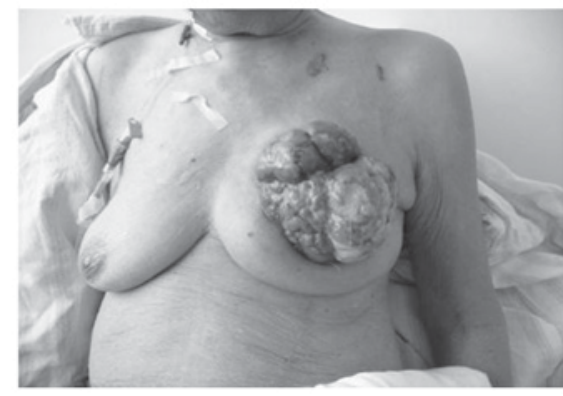

Figure 1. Case 1: An 83-year-old female with neuroendocrine carcinoma The tumor had a cauliflower-like appearance and was $15 \times 15 \mathrm{~cm}$ in diameter.

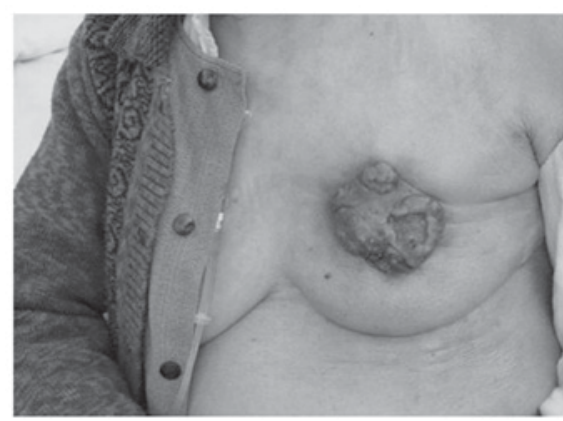

Figure 2. Case 1: Following 3 cycles of intra-arterial interventional therapy, the tumor decreased to a diameter of $5 \times 4 \mathrm{~cm}$.

Case 3. A 46-year-old woman visited the Department of Breast Surgery, People's Liberation Army Army General Hospital in March 2014. In 2012, the patient suffered an injury to the right breast and subsequently noticed that there was a $4-\mathrm{cm}$ lump above the nipple. In February 2013, the patient attempted to reduce the size of the lump by covering it with Chinese herbs, but itcontinued to grow. The patient subsequently attempted treatment with traditional Chinese medicines, but the lump continued to grow rapidly to a diameter of $20 \times 20 \mathrm{~cm}$ and the skin became darker (Fig. 6). Upon examination, a 5-cm axillary lymph node was observed and biopsy revealed an invasive carcinoma that was ER-positive (90\%), PR-positive (3-5\%), Ki-67-positive (40\%) and HER2-negative. The patient received 5 cycles of a doxorubicin, epirubicin and cyclophosphamide neoadjuvant chemotherapy regimen and the tumor became softer, with a decreased diameter of $10 \times 10 \mathrm{~cm}$, without any palpable axillary lymph nodes. The previously darkened skin became lighter, began to peel and eventually returned to an almost normal color (Fig. 7). IAIT with $40 \mathrm{mg}$ pirarubicin, $30 \mathrm{mg}$ hydroxycamptothecin and 1,000 mg fluorouracil was administered via the left internal thoracic artery. IAIT and embolization were conducted simultaneously during the second treatment cycle and the tumor continued to become softer and smaller (Fig. 8). In August 2014, the patient underwent a modified radical mastectomy and partial pectoralis-major removal. Residual invasive carcinoma was observed in the pathological sample and $8 / 8$ axillary lymph nodes exhibited invasion. Immunohistochemistry revealed that the tumor was ER-positive (90\%), PR-positive (5-10\%), Ki-67-positive (10\%) and HER2-negative. The patient recovered normally without any complications. Subsequently, 6 cycles of gemcitabine

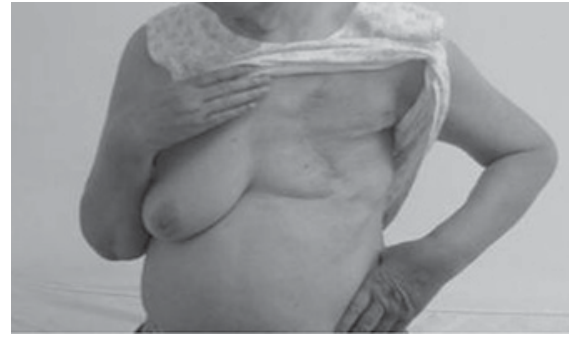

Figure 3. Case 1: Postoperative view of the 90 -year-old female patient following a modified radical mastectomy.

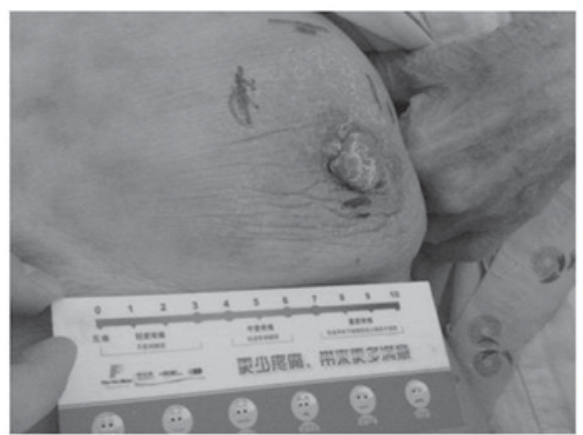

Figure 4. Case 2: A 90-year-old female with invasive ductal carcinoma. The tumor was $6 \mathrm{~cm}$ in diameter with a 16x15-cm area of surrounding red skin.

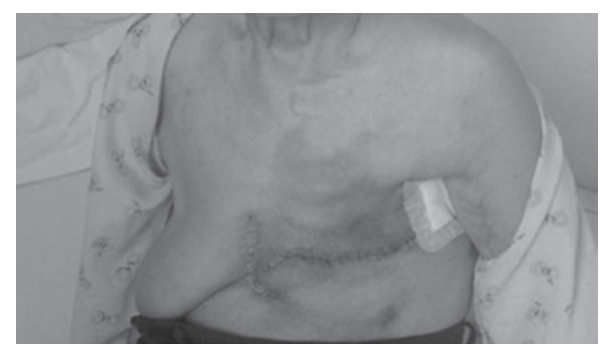

Figure 5. Case 2: Postoperative view of the patient following 4 cycles of intra-arterial interventional therapy and a modified radical mastectomy.

$(1,500 \mathrm{mg}$ on days 1,8$)$ and capecitabine $(2,000 \mathrm{mg}$ twice daily on days 1-14) in combination with goserelin (3.6 mg once per month) were administered, and radiotherapy (50 Gy/5 weeks) followed and then endocrinotherapy (exemestane $25 \mathrm{mg}$ once daily) are ongoing (Fig. 9).

Case 4. A 58-year-old woman visited the Department of Breast Surgery, People's Liberation Army Army General Hospital in September 2014. In September 2003, the patient had been diagnosed with right invasive breast cancer and underwent a modified radical mastectomy. Immunohistochemistry revealed that the tumor was ER-negative, PR-negative, Her-2-negative and Ki-67-positive (40\%), and no lymph nodes exhibited invasion $(0 / 25)$. The patient received one cycle of pirubicin $(6 \mathrm{mg}$ on days 1,8$)$ and fluorouracil $(750 \mathrm{mg}$ on days 1,8$)$, three cycles of cyclophosphamide (800 mg on days 1,8), epirubicin (90 mg on day 1) and fluorouracil $(750 \mathrm{mg}$ on days 1,8$)$ and one cycle of epirubicin (90 mg on day 1) and paclitaxel $(270 \mathrm{mg}$ on day 1) chemotherapy. In 2010, the patient found a tumor in the left breast, but did not obtain a diagnosis or receive any 


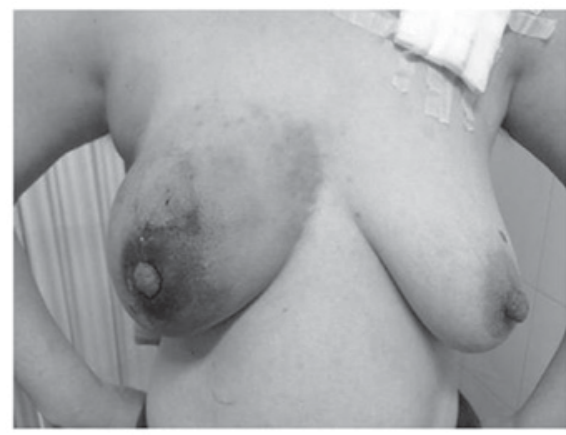

Figure 6. Case 3: A 46-year-old female with invasive carcinoma. The tumor was $20 \times 20 \mathrm{~cm}$ in diameter with dark skin.

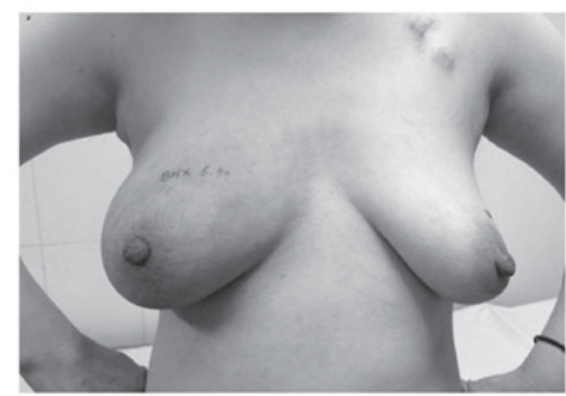

Figure 7. Case 3: Following 5 cycles of a doxorubicin, epirubicin and cyclophosphamide regimen, the tumor was $10 \times 10 \mathrm{~cm}$ in diameter without any palpable axillary lymph nodes. The skin color had almost normalized.

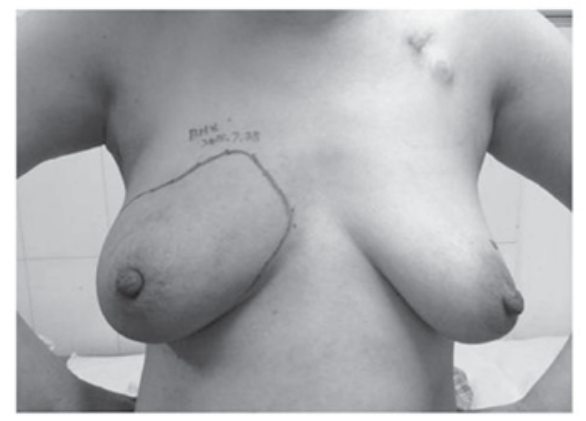

Figure 8. Case 3: Following 2 cycles of intra-arterial interventional therapy, the tumor continued to become softer and smaller.

treatment. In 2013, the tumor grew larger, red, swollen, painful and ulcerated. Biopsy revealed a diagnosis of invasive ductal carcinoma and immunohistochemistry revealed that the tumor was ER-negative, ER $\beta$-positive (70\%), Her-2-negative and Ki-67-positive (85\%). Since December 2013, the patient underwent 1 cycle of capecitabine $(1,500 \mathrm{mg}$ twice daily on days 1-14) followed by 2 cycles of capecitabine (1,500 mg twice daily on days 1-14) and docetaxel (120 mg on day 1), but the tumor grew larger. Following one cycle of adriamycin liposome (60 mg on day 1), two cycles of adriamycin liposome (40 mg on day 1) and cyclophosphamide (1,000 mg on day 1), one and a half of gemcitabine $(1,500 \mathrm{mg}$ on days 1,8$)$ and carboplatin (600 mg on day 1) chemotherapy the tumor continued to grow. When the patient visited the Department of Breast Surgery, People's Liberation Army Army General Hospital, the tumor

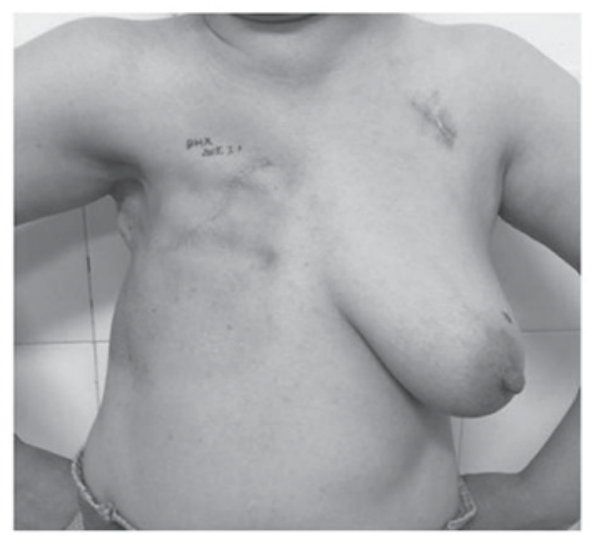

Figure 9. Case 3: Postoperative view of the patient following a modified radical mastectomy and partial pectoralis-major removal.

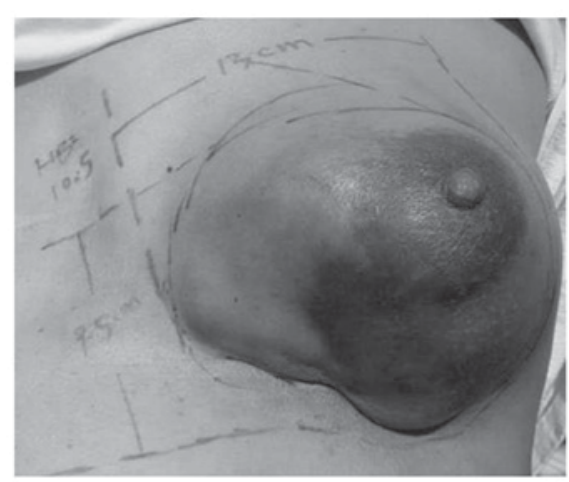

Figure 10. Case 4: A 58-year-old female with invasive ductal carcinoma. The tumor was $13 \times 9.5 \mathrm{~cm}$ in diameter.

was $13 \times 9.5 \mathrm{~cm}$ in diameter (Fig. 10). Biopsy revealed a diagnosis of invasive ductal carcinoma and immunohistochemistry demonstrated that the tumor was ER-negative, PR-negative, Ki-67-positive (85\%) and HER2-negative. On September 23, 2014, DSA demonstrated that the blood supply to the tumor was from the left internal thoracic artery and a branch of the left axillary artery. Subsequently, $40 \mathrm{mg}$ lobaplatin, $30 \mathrm{mg}$ hydroxycamptothecin and $40 \mathrm{mg}$ epirubicin was administered via the left internal thoracic artery and a branch of the left axillary artery. The ratio of medication administered via the two vessels was 4:1. Intra-arterial embolization with $1.5-\mathrm{mm}$ selective absorbable gelatin sponge particles was conducted via the left internal thoracic artery. Following the first cycle of IAIT, the tumor became slightly softer and smaller (Fig. 11). For the second cycle of IAIT, the treatment was composed of $150 \mathrm{mg}$ etoposide and $40 \mathrm{mg}$ navelbine. A total of $80 \%$ of the medication was administered via the left internal thoracic artery, while the remaining $20 \%$ was administered via a branch of the left axillary artery. Intra-arterial embolization was also performed and the tumor continued to become softer and smaller. For the third cycle of IAIT, $135 \mathrm{mg}$ etoposide, $30 \mathrm{mg}$ navelbine and $30 \mathrm{mg}$ epirubicin was administered via the left internal thoracic artery and the left external thoracic artery. Following the third cycle of IAIT, the lower half of the tumor became necrotic and fell off (Fig. 12). On September 25 2014, the patient underwent a modified radical mastectomy 


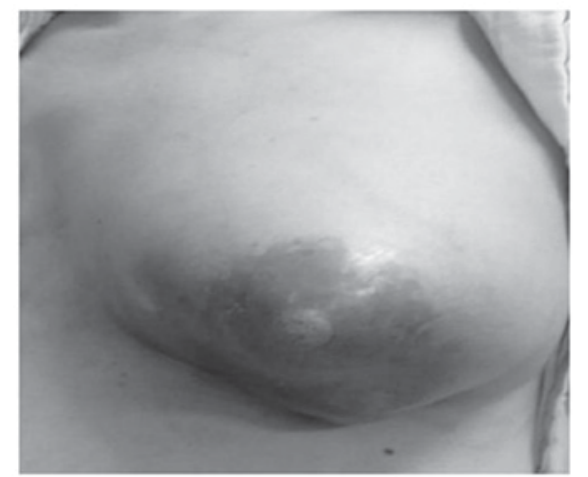

Figure 11. Case 4: Following the first cycle of intra-arterial interventional therapy, the tumor became slightly softer and smaller.

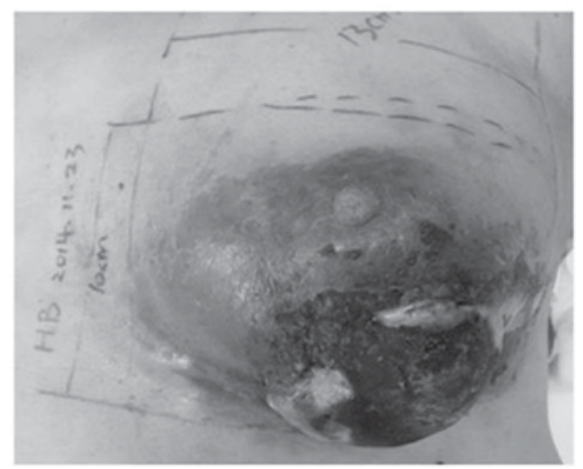

Figure 12. Case 4: Following 3 cycles of intra-arterial interventional therapy, the lower half of the tumor became necrotic and fell off.

with removal of a portion of the pectoralis-major (Fig. 13). Pedicled latissimusdorsi was harvested to repair a defect of the chest wall . The patient recovered normally one week later (Fig. 14). Pathology revealed a grade III invasive ductal carcinoma that was ER-negative, PR-negative, Ki-67-positive (50\%) and HER2-negative. One axillary lymph node (1/15) and no subclavian lymph nodes (0/4) were invaded by the carcinoma. Following 1 cycle of adjuvant chemotherapy with etoposide (140 $\mathrm{mg}$ on days 1 to 5 ) and navelbine (50 $\mathrm{mg}$ on days 1,8), a gynecological tumor was identified and the uterus and bilateral uterine adnexa were removed on January 13, 2015. Pathology revealed serous carcinoma of the right fallopian tube. Local radiotherapy (50 Gy/5 weeks) was performed between January 29 and March 6, 2015. The radiation range covered the chest wall, subclavicular and supraclavicular area (Fig. 15). Another cycle of adjuvant chemotherapy with etoposide $(120 \mathrm{mg}$ on days 1 to 5) and navelbine (40 mg on days 1,8) was completed in April 2015. Dermis and pleural fluid invasion of the carcinoma was identified in May, but despite treatment with paclitaxel liposome, the patient succumbed to gynecological tumor in June 2015.

Patient evaluation. Liver, kidney, bone marrow and cardiac function was assessed prior to and following IAIT. Clinical observations included the size, color and mobility of the tumor 3 weeks after IAIT. If the size of the tumor decreased notably and the involved skin and muscle improved markedly, the patients underwent surgical resection. Otherwise,

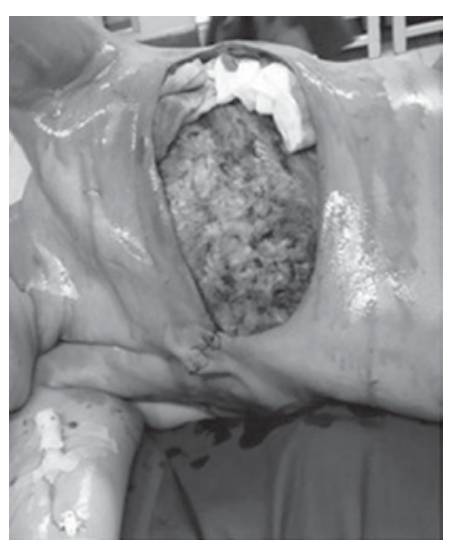

Figure 13. Case 4: Modified radical mastectomy was performed with removal of a portion of the pectoralis-major.

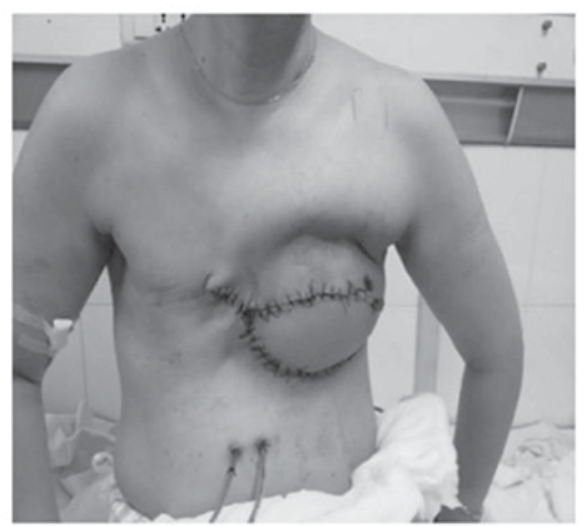

Figure 14. Case 4: One week postoperative view of the patient after the chest wall defect was repaired with pedicled latissimusdorsi.

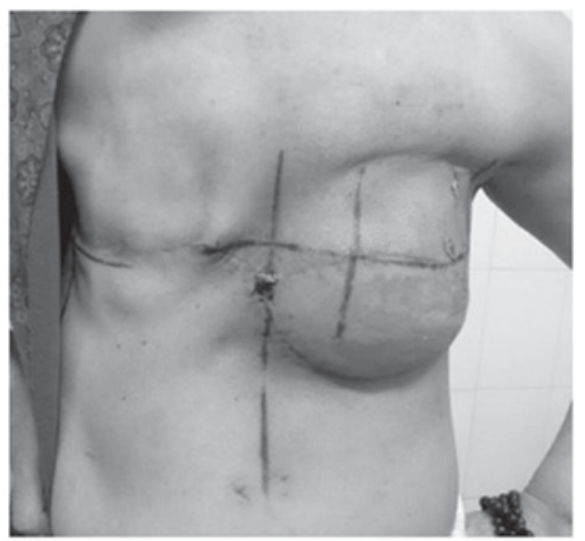

Figure 15. Case 4: Postoperative local radiotherapy following the observation of a gynecological tumor and the removal of the uterus and bilateral uterine adnexa. Postoperative radiation range of the patient covered the chest wall, subclavicular and supraclavicular area.

the patients underwent another cycle of IAIT. Efficacy of the IAIT was assessed using the UICC criteria (6). A complete response (CR) was defined as the complete disappearance of all clinical evidence of the tumor and enlarged lymph nodes by clinical evaluation, mammogram and ultrasound. A partial response was defined as a $\geq 50 \%$ decrease in the two longest 
perpendicular diameters of the tumor mass and local lymph nodes. Mild response was defined as a $\leq 50 \%$ decrease in the two longest perpendicular diameters of the tumor mass and local lymph nodes. Stable disease (SD) was defined as no change in the two longest perpendicular diameters of the tumor mass and local lymph nodes. Progressive disease (PD) was defined as the appearance of any new lesions not previously identified or an increase in the two longest perpendicular diameters of the tumor mass and local lymph nodes.

\section{Results}

All 7 patients were female and aged between 34 and 90 (mean age, 59). The largest observed lesion was $20 \mathrm{~cm}$ in diameter. Overall, 5 patients exhibited palpable lymph nodes and 6 had received $\geq 1$ cycle of neoadjuvant chemotherapy. None of the patients exhibited any distant metastatic disease, but mild myelosuppression occurred in 2 patients. No clear side effects, including cardiac failure and normal skin necrosis, were observed at any point. PR occurred in 6 patients and SD occurred in 1 patient. All the patients underwent surgical resection following IAIT. A total of 4 of the patients were diagnosed with triple-negative breast cancer. Progression-free survival (PFS) time ranged between 6 and 88 months. In total, 1of the patients succumbed as a result of another disease 8 months after receiving IAIT, another succumbed from carcinoma of the right fallopian tube and multiple organ metastases 9 months after IAIT and another survived for 11 months and died of heart disease. The other 4 patients remain alive and no local recurrence or metastasis has been observed.

\section{Discussion}

Breast cancer is the most frequently occurring type of cancer among females, the leading cause of cancer-associated mortality and one of the main causes of morbidity and mortality for females worldwide (7). LABC includes large breast tumors, tumors involving the skin over the breast or the underlying chest wall muscles, and breast cancer with extensive local lymph node involvement. LABC also includes inflammatory breast cancer, a rapidly proliferating cancer type that results in a red and swollen breast appearance. LABC accounts for $5-15 \%$ of new breast cancer cases in the USA and for $40-60 \%$ of new cases in non-industrialized countries (8). LABC is generally treated with combined-modality therapy that includes systemic chemotherapy, surgery, radiotherapy, endocrinotherapy and targeted therapy (9).

Neoadjuvant chemotherapy is of paramount importance in the treatment of breast cancer and is considered to reduce tumor size, thereby rendering breast-conserving surgery possible in LABC (10-12). Anthracycline combined with taxane is the standard treatment for LABC and $84 \%$ of the previously inoperable cases may become resectable upon its use (13). A pathological CR (PCR) is easy to obtain in early-stage breast cancer patients. However, in LABC, only a small proportion of patients may achieve PCR due to the large tumor size (14). Additionally, re-administration of anthracycline to patients with anthracycline-pretreated breast cancer may be associated with reduced efficacy and significant cardiotoxicity (15), making LABC treatment a challenge to clinical physicians and surgeons. The present study indicates that IAIT is an option for the management of older patients with LABC, particularly those with cardiac dysfunction. In the present study, the eldest patient was 90 years old and was unable to tolerate even 1 cycle of paclitaxel liposome neoadjuvant chemotherapy. However, the patientwas able to complete four cycles of IAIT without any observable side effects.

The breast is a solid organ with a definite blood supply from the internal thoracic artery, lateral thoracic artery, intercostal arteries and subscapular artery (16). IAIT is not a standard therapy, but is occasionally performed with the aim of downstaging or for local control. There are a number of studies regarding the use of IAIT (17-20). Shimamoto et al (20) reported that the local response rate of redistributed subclavian arterial infusion chemotherapy for LABC was $77.3 \%$, and that the response rate was higher $(83.3 \%)$ if the first treatment was infusion chemotherapy. In the present study, the response rate was $85.7 \%$ (6/7). IAIT provides a higher plasma concentration, better clinical results and fewer side effects than traditional systemic chemotherapy $(21,22)$. A previous study demonstrated that IAIT is effective and safe for the treatment of locally advanced breast cancer even in elderly patients (19). In the present study, none of the patients experienced any serious side effects, including normal skin necrosis, liver, kidney or cardiac failure, and all of the patients completed IAIT.

One study observed that, compared with that of early-stage breast cancer, the overall survival (OS) rate of LABC was lower, and the risk of local recurrence and metastasis was markedly higher, with a 10-year survival rate of $\sim 30 \%$ (23). Hoehne et al (24) reported that the 5-year OS rate for stage IIIC LABC was $71.9 \%$. In China, the 5-year OS rate for stage IIB and III LABC was reported as $71.3 \%$ (25). In the present study, the OS data are not complete as a number of patients remain alive, but the current longest OS time is 88 months.

Endocrinotherapy is also an effective treatment for LABC, particularly for patients with the lumina A subtype. However, if the tumor burden is heavy (diameter $>10 \mathrm{~cm}$ ), good control may not be achieved in a short time period. If the tumor is not responsive to endocrinotherapy and metastasizes to other organs, the outcome is generally poor. The principle of the treatment of LABC is to rapidly control and decrease the tumor size, reduce the tumor burden, gain opportunity for surgical resection, decrease drug resistance and increase therapeutic efficacy. For large lumina A LABC, IAIT may be used to rapidly downstage and decrease tumor burden. In the present study, case 1 is a good example of such large lumina A patient, as this patient needs to rapidly reduce the tumor size. The patient was 83 years old with severe anemia and heart failure. Chemotherapy and surgical resection was not suitable due to the size of the tumor and the poor physical condition of the patient. As a result of IAIT, the tumor size was rapidly decreased, thereby enabling surgical resection. The PFS of this patient is currently 88 months and the patient is now 90 years old. For older patients with large tumors who are not suitable for neoadjuvant chemotherapy, local IAIT with or without embolization is a safe and effective method without any clear side effects.

Timing is extremely important in the management of LABC. Due to the fact that a number of patients experience bleeding, anemia and hypoproteinemia, there may not be much 
opportunity to attempt alternative neoadjuvant chemotherapy regimens. Particularly with regard to precision medicine, the manner in which to precisely identify target patients and make good treatment choices has become a challenge. Improved understanding of tumor subtypes and genetic tests may inform clinicians that certain patients may not respond to neoadjuvant chemotherapy, thereby indicating the use of IAIT to control the disease.

The present study has a number of limitations. To begin with, there were not enough cases for statistical analysis to be possible. Additionally, the present study was retrospective and therefore was not strictly a case-control study. Furthermore, the drugs used for IAIT were not uniform due to differences in tumor subtypes. However, all the tumors were large and the clinical effects were clear, thereby permitting the conclusion that IAIT is a safe and effective approach for the treatment of LABC.

\section{References}

1. Igene H: Global health inequalities and breast cancer: An impending public health problem for developing countries. Breast J 14: 428-434, 2008.

2. Veronesi U, Boyle P, Goldhirsch A, Orecchia R and Viale G: Breast cancer. Lancet 365: 1727-1741, 2005.

3. Siegel R, Ma J, Zou Z and Jemal A: Cancer statistics, 2014. CA Cancer J Clin 64: 9-29, 2014.

4. Ziegler RG, Anderson WF and Gail MH: Increasing breast cancer incidence in China: The numbers add up. J Natl Cancer Inst 100: 1339-1341, 2008.

5. Gradishar WJ, Anderson BO, Balassanian R, Blair SL, Burstein HJ, Cyr A, Elias AD, Farrar WB, Forero A, Giordano SH, et al: Breast Cancer Version 2. 2015. J Natl Compr Canc Netw 13: 448-475, 2015.

6. Miller AB, Hoogstraten B, Staquet M and Winkler A: Reporting results of cancer treatment. Cancer 47: 207-214, 1981.

7. Toriola AT and Colditz GA: Trends in breast cancer incidence and mortality in the United States: Implications of prevention. Breast Cancer Res Treat 138: 665-673, 2013.

8. Winer EP, Mrrow M, Osborne CK and Harris JR: Malignant tumors of the breast. In: De Vita T, Hellman JS and Rosenberg SA eds. Cancer, Principle and Practice of Oncology, 6th Edition. Philadelphia: Lippincott Williams \& Wilkins, pp. 1697, 2001.

9. Kaufmann M, von Minckwitz G, Bear HD, Buzdar A, McGale P, Bonnefoi H, Colleoni M, Denkert C, Eiermann W, Jackesz R, et al: Recommendations from an international expert panel on the use of neoadjuvant (primary) systemic treatment of operable breast cancer: New perspectives 2006. Ann Oncol 18: 1927-1934, 2007.

10. Uzzan B, Nicolas P, Cucherat M and Perret GY: Microvessel density as a prognostic factor in women with breast cancer: A systematic review of the literature and meta-analysis. Cancer Res 64: 2941-2955, 2004.

11. Schwartz GF and Hortobagyi GN: Proceedings of the consensus conference on neoadjuvant chemotherapy in carcinoma of the breast, April 26-28, 2003, Philadelphia, Pennsylvania. Cancer 100: 2512-2532, 2004.

12. Bevers TB, Anderson BO, Bonaccio E, Buys S, Daly MB, Dempsey PJ, Farrar WB, Fleming I, Garber JE, Harris RE, et al: NCCN clinical practice guidelines in oncology: Breast cancer screening and diagnosis. J Natl Compr Canc Netw 7: 1060-1096, 2009.
13. Alvarez RH, Booser DJ, Cristofanilli M, Sahin AA, Strom EA, Guerra L, Kau SW, Gonzalez-Angulo AM, Hortobagyi GN and Valero V: Phase 2 trial of primary systemic therapy with doxorubicin and docetaxel followed by surgery, radiotherapy, and adjuvant chemotherapy with cyclophosphamide, methotrexate, and 5-fluorouracil based on clinical and pathologic response in patients with stage IIB to III breast cancer: Long-term results from the University of Texas M. D. Anderson Cancer Center Study ID97-099. Cancer 116: 1210-1217, 2010.

14. Nahleh Z, Sivasubramaniam D, Dhaliwal S, Sundarajan V and Komrokji R: Residual cancer burden in locally advanced breast cancer: A superior tool. Curr Oncol 15: 271-278, 2008.

15. Pacilio C, Morabito A, Nuzzo F, Gravina A, Labonia V, Landi G, Rossi E, De Maio E, Di Maio M, D'Aiuto G, et al: Is epirubicin effective in first-line chemotherapy of metastatic breast cancer (MBC) after an epirubicin-containing adjuvant treatment? A single center phase III trial. Br J Cancer 94: 1233-1236, 2006.

16. Bilbao JI, Rebollo J, Longo JM, Mansilla F, Muñoz-Galindo L and Vieitez JM: Neoadjuvant intra-arterial chemotherapy in inflammatory carcinoma of the breast. Br J Radiol 65: 248-251, 1992.

17. Murakami M, Kuroda Y, Nishimura S, Sano A, Okamoto Y, Taniguchi T, Nakajima T, Kobashi Y and Matsusue S: Intraarterial infusion chemotherapy and radiotherapy with or without surgery for patients with locally advanced or recurrent breast cancer. Am J Clin Oncol 24: 185-191, 2001.

18. Kitagawa K, Yamakado K, Nakatsuka A, Tanaka N, Matsumura K, Takeda K, Kawarada Y: Preoperative transcatheter arterial infusion chemotherapy for locally advanced breast cancer (stage IIIb) for down-staging and increase of resectability. Eur J Radiol 43: 31-36, 2002.

19. Pacetti P, Mambrini A, Paolucci R, Sanguinetti F, Palmieri B, Della Seta R, Muttini MP, Fiorentini G and Cantore M: Intra-arterial chemotherapy: A safe treatment for elderly patients with locally advanced breast cancer. In Vivo 20: 761-764, 2006.

20. Shimamoto H, Takizawa K, Ogawa Y, Yoshimatsu M, Yagihashi K, Okazaki H, Kanemaki Y, Nakajima Y, Ohta T, Ogata $\mathrm{H}$ and Fukuda M: Clinical efficacy and value of redistributed subclavian arterial infusion chemotherapy for locally advanced breast cancer. Jpn J Radiol 29: 236-243, 2011.

21. Fiorentini G, Tsetis D, Bernardeschi P, Varveris C, Rossi S, Kalogeraki A, Athanasakis E, Dentico P, Kanellos P, Biancalani M, et al: First-line intraarterial chemotherapy (IAC) with epirubicin and mitoxantrone in locally advanced breast cancer. Anticancer Res 23: 4339-4345, 2003.

22. Takatsuka Y, Yayoi E, Kobayashi T, Aikawa T and Kotsuma Y: Neoadjuvantintraarterial chemotherapy in locally advanced breast cancer: A prospective randomized study. Osaka Breast Cancer Study Group. Jpn J Clin Oncol 24: 20-25, 1994.

23. Mathew J, Asgeirsson KS, Cheung KL, Chan S, Dahda A and Robertson JF: Neoadjuvant chemotherapy for locally advanced breast cancer: A review of the literature and future directions. Eur J Surg Oncol 35: 113-122, 2009.

24. Hoehne F, Chen S, Mabry H and Giuliano AE: An update on prognosis in breast cancer patients with extensive axillary disease. Breast J 14: 76-80, 2008.

25. Huang O, Chen C, Wu J, Chen S, Chen X, Liu G, Hu Z, Lu J, Wu J, Shao Z, et al: Retrospective analysis of 119 Chinese noninflammatory locally advanced breast cancer cases treated with intravenous combination of vinorelbine and epirubicin as a neoadjuvant chemotherapy: A median follow-up of 63.4 months. BMC Cancer 9: 375, 2009. 